\title{
PROPAGAÇÃO DE ERROS E INCERTEZAS EM EXPERIMENTOS
}

Erlon Lopes PEREIRA ${ }^{1}$

Bárbara Emerick DIAS ${ }^{2}$

Bianca Nunes LERIS ${ }^{3}$

Joyce de Souza TANURE ${ }^{4}$

${ }^{1}$ Departamento de Química. Setor de Engenharia Química. Universidade Federal de Viçosa. Campus: Viçosa-
MG. CEP: 36570-900. e-mail: erlon.pereira@ufv.br/erlonlopes@gmail.com.

${ }^{2}$ Universidade Federal de Viçosa/Departamento de Química. barbara.emerick@ufv.br

${ }^{3}$ Universidade Federal de Viçosa/Departamento de Química. biancaleris@ hotmail.com

${ }^{4}$ Universidade Federal de Viçosa/Departamento de Química. joyce.tanure@ufv.br

\section{RESUMO:}

No decorrer de uma análise industrial, devem ser considerados inúmeros fatores que influenciam no modo como o trabalho será executado. Entre eles, na parte quantitativa dos procedimentos, estão os erros e incertezas. Erro é uma palavra empregada para designar a diferença entre um valor experimental e o seu valor verdadeiro. Por outro lado, a incerteza é uma estimativa que quantifica a confiabilidade do resultado de uma medição. Enquanto no erro existe uma dependência do conhecimento do valor referencial do que se está medindo, a incerteza pode ser calculada sem tal valor. Outro conceito a ser avaliado é o de propagação de incertezas, que é justamente a repercussão de vários valores duvidosos. Para calcular e minimizar esse quesito, é utilizado o software Engeneering Equation Solver, programa que facilita os cálculos e fornece um bom parâmetro dos resultados.

Palavras-chave: Software, Modelagem matemática, Instrumentação, Exatidão.

\begin{abstract}
:
In the course of an industrial analysis, several factors, which play important role in the way the work is conducted, must be taken into account. Among those, in the quantitative part of the procedures, there are the errors and uncertainties. Error is the term employed in order to indicate the difference between an experimental value and its actual value. On the other hand, uncertainty is an estimation that quantifies the reliability of a measurement result. Whereas in the error there is a dependence on the reference value of the measurement, the uncertainty can be calculated without such value. Another concept to be evaluated is the propagation of uncertainty, which is the repercussion caused by various doubtful values. In order to calculate and minimize this factor we utilize the Engineering Equation Solver, a software that facilitate the calculations and provide a good parameter of the results.

Keywords: Software, mathematical modeling, instrumentation, Accuracy
\end{abstract}

Recebido em: 05/07/2016 - Aprovado em: 11/09/2016 - Disponibilizado em: 18/12/2016 


\section{INTRODUÇÃO}

A metrologia está contida nas mais diversas atividades e itens presentes na rotina da população, visto que engloba sistemas de medição sendo parte fundamental de todo sistema de gestão da qualidade (BARATTO et al., 2008).

Silva Neto (2012) define metrologia como a ciência das medidas e suas aplicações abrangendo todos os aspectos teóricos e práticos que asseguram a precisão exigida no processo produtivo. A utilização dessa ciência visa à garantia da qualidade de produtos e serviços através de um estudo detalhado dos instrumentos envolvidos na produção, sejam eles digitais ou analógicos. Desse modo, cabe ao executor das operações uma escolha, assim como uma adaptação às circunstâncias produtivas.

De acordo com Santos Júnior e Irigoyen (1985), dados experimentais nunca terão precisão e exatidão absoluta, porém, alguns dados são mais precisos (ou exatos) do que outros, e é necessário estabelecer uma medida que permita verificar quão bom é o valor da medição.Em soma, esses fatores merecem uma atenção especial, na medida em que averiguam o quão bom é o resultado de uma determinada medida.

O trabalho de um profissional responsável por um serviço ou cadeia produtiva, envolve muitas vezes rotas matemáticas e manuseios de aparelhos industriais que apresentam características singulares.

Neste contexto o erro e a incerteza são conceitos que estão diretamente relacionados ao aspecto quantitativo de uma análise. Ao realizar um experimento ou marchas de cálculo, para aquantificação da propagação de erros e incertezas é necessário estudá-los através de processos matemáticos.Dessa forma é fundamental o entendimento dos conceitos de metrologia como os que serão discutidos ao longo do artigo. Visto o exposto este trabalho teve como objetivo apresentar uma os conceitos que envolvem os cálculos de incerteza em experimentos de engenharia, bem como apresentar exemplos que explicam de forma didática os procedimentos padrões exigidos nos protocolos de controle de qualidade e reprodutibilidade de análises experimentais.

\section{DESENVOLVIMENTO TEÓRICO}

\subsection{ERROS}

Segundo Santos Júnior e Irigoyen (1985), o valor verdadeiro de uma grandeza física experimental pode ser considerado o objetivo final do processo de medição e é entendido como problemaporque esse valor é sempre desconhecido. As únicas grandezas que têm seus valores verdadeiros conhecidos exatamente são aquelas que não dependem de 
dados experimentais para serem determinadas, tal como o número pi $(\pi)$.

De acordo com Vuolo (2005), se $y_{v}$ é o valor verdadeiro de um mensurando e $y$ é o resultado de uma medição, o erro $(\eta)$ em $y$ é definido pela Equação 1.

$$
\eta=y-y_{v}
$$

Os diferentes tipos de erros podem ser agrupados em 2 grandes grupos que são os erros sistemáticos e os erros estatísticos (aleatórios). Erros estatísticos resultam de variações aleatórias no resultado da medição, devido a fatores que não podem ser controlados ou que, por qualquer motivo, não são controlados. Em geral, estas variações se devem somente ao processo de medida. Por exemplo, na medição de massa com uma balança, correntes de ar podem introduzir este tipo de erro, que pode ser reduzido ou praticamente eliminado colocandose a balança em uma caixa de vidro ou mesmo em vácuo.

Vuolo (2005) define que a expressão "erro praticamente eliminado" significa que este foi reduzido de forma a se tornar muito menor que os demais erros envolvidos na medição. Em geral, um erro não pode ser eliminado, mas apenas reduzido.Uma solução para reduzir os erros estatísticos consiste em repetir muitas vezes a medição, uma vez que o valor médio de um grande número de resultados tem erro estatístico menor.
Segundo Vuolo (2005) o erro sistemático é o mesmo para qualquer resultado quando a medição é repetida.São erros constantes ou que variam sob uma determinada lei e podem ter causas muito diversas e geralmente se enquadram em um dos tipos definidos como instrumentais, ambientais, observacionais, teóricos e grosseiros.

Mills (2004) define erros sistemáticos instrumentais como erros que resultam da calibração do instrumento de medição. Além do erro na calibração inicial do instrumento, deve ser observado que diversos fatores podem influenciá-la como temperatura, alteração das características dos materiais e desgaste de partes móveis. Este tipo de erro pode ser reduzido por meio da recalibração ou nova aferição do instrumento de medida e correção dos resultados.

Erros sistemáticos ambientais são devido a efeitos do ambiente sobre a experiência. Fatores ambientais como temperatura, pressão, umidade, luz, aceleração da gravidade podem introduzir erro nos resultados de uma medição. Tal tipo de erro pode ser reduzido ou praticamente eliminado se as condições ambientais forem conhecidas e, de preferência, controladas. Segundo, em qualquer processo de medição é importante registrar todas as grandezas ambientais que possam influir na prática experimental (VUOLO, 2005).

Erros sistemáticos observacionais são erros devido a pequenas falhas de 
procedimento ou limitações do próprio observador. Este erro ocorre devido ao efeito de paralaxe na leitura de escalas de instrumentos (efeito devido ao não alinhamento correto entre o olho do observador, o indicador da leitura e a escala do instrumento). Erros sistemáticos observacionais podem ser reduzidos seguindose cuidadosamente os procedimentos corretos para uso dos instrumentos (VUOLO, 2005).

Erro sistemático teórico é o erro que resulta do uso de fórmulas teóricas aproximadas para obtenção de resultados e podem ser reduzidos utilizando-se modelos físicos, formulas e valores para as constantes suficientemente exatas para o fenômeno em questão (VUOLO,2005).

Erros grosseiros são enganos que podem ocorrer na medição ou nos cálculos, e, portanto, não são considerados erros do ponto de vista da teoria dos erros descrita por Vuolo (2005). Para que os erros grosseiros sejam evitados, as medições devem ser repetidas e conferidas cuidadosamente. Também se deve analisar criteriosamente a consistência dos resultados experimentais, com base em métodos estatísticos.

Em metrologia, o conceito de exatidão (ou acuidade) refere-se ao grau de concordância de uma medida com seu valor alvo. Ou seja, quanto mais próxima do valor verdadeiro correspondente, mais exata é a medida. $\mathrm{O}$ conceito de precisão (fidedignidade ou reprodutibilidade), em contrapartida, refere-se somente ao grau de dispersão da medida quando repetida sob as mesmas condições. Em outras palavras, uma medida é precisa se, repetida diversas vezes, apresentar resultados semelhantes. Santos Júnior e Irigoyen (1985) como exatidão e precisão são qualidades bastante diferentes, é possível que o resultado de uma medição seja exato e preciso, exato e impreciso, inexato e preciso ou inexato e impreciso.

\subsection{INCERTEZAS}

A Incerteza de medição é um parâmetro associado ao resultado de um procedimento, que caracteriza a dispersão de valores atribuídos à grandeza submetida à tal ensaio (CAMPOS et al., 2008).

Assim, por definição, a incerteza é um valor que estima o quanto um resultado é confiável. Quando maior a incerteza, menor a confiabilidade desse resultado. Ao mesmo tempo, é plausível apontar que incerteza e erro são dois parâmetros diferentes. Enquanto o estudo do erro está relacionado à necessidade de conhecer o valor verdadeiro do que está sendo medido, o cálculo da incerteza não depende desse tipo de análise.

A incerteza é um conceito mais instrumental e mais aplicável que o conceito de erro. De acordo com Campos et al. (2008) há dois tipos de incerteza, que se diferenciam por serem calculadas por procedimentos 
distintos. No primeiro grupo de procedimentos, estão todos os métodos envolvendo análise de uma série de observações da mesma grandeza física. Ocorre quando o desvio padrão da média dessa série de observações é calculado. No outro grupo, as avaliações são utilizadas quando é difícil realizar repetidas observações (SANTOS JÚNIOR e IRIGOYEN; 1985).

Ao avaliar a incerteza propagada de um experimento, é necessário considerar as incertezas provenientes de diversas fontes, como erros de leitura em instrumentos analógicos, realização imperfeita do procedimento de ensaio, amostragem, variações em repetidas observações sob condições aparentemente idênticas, entre outras (BARATTO et al., 2008).

De acordo com Vuolo (2005), a incerteza em um resultado pode ser especificada de diferentes maneiras, sendo que as mais usuais são a incerteza padrão, incerteza expandida com confiança, limite de erro e erro provável.

A incerteza padrão é definida como o desvio padrão da distribuição de erros. É a maneira mais usada para indicar a incerteza em procedimentos experimentais. Enquanto isso, a incerteza expandida com confiança é um múltiplo desse desvio padrão.

O limite de erro, adentrando nos conceitos também definidos previamente, é um valor máximo admissível para o erro. E o erro provável é o valor que tem uma probabilidade definida de ser excedido pelo erro. Porém, essa forma não é mais utilizada.

Paralelamente à definição de incerteza, deve ocorrer também uma exposição do conceito de propagação da mesma. Como toda grandeza de entrada em um processo de cálculo apresenta uma incerteza, o mesmo deve ocorrer com as grandezas de saída. A repercussão de incertezas entre as grandezas de entrada e saída é justamente a propagação de incertezas, a qual pode ser definida por uma lei, como mostrado nas equações 2 e 3 .

$$
\begin{aligned}
& R=R\left(X_{1}, X_{2}, X_{3}, \ldots, X_{n}\right) \\
& \omega_{\mathrm{R}}=\left[\left(\left(\frac{\partial \mathrm{R}}{\partial \mathrm{x} 1} \times \omega_{1}\right)^{2}+\cdots\right.\right. \\
& \left.\left.\quad+\left(\frac{\partial \mathrm{R}}{\partial \mathrm{xn}} \times \omega_{\mathrm{n}}\right)^{2}\right)\right]^{1 / 2}
\end{aligned}
$$

Em que:

$\omega_{\mathrm{R}}$ : é a propagação da incerteza;

$\omega_{\mathrm{N}}$ : é a incerteza de cada grandeza utilizada;

$\frac{\partial R}{\partial X n}$ : é a grandeza que está sendo calculada, em função da grandeza utilizada.

Analisando a Equação 3 é possível observar que a grandeza que mais influenciará no valor final de $\omega_{\mathrm{R}}$ será a com o maior valor de derivada em relação a uma das variáveis, ou seja, $\frac{\partial R}{\partial x}$. Consequentemente, ações para minimizar a incerteza total podem envolver medidas que promovam uma diminuição desses termos. 


\subsection{ENGINEERING EQUATION SOLVER (EES)}

Para resolver a maioria dos problemas envolvendo incertezas e propagação de incertezas, utiliza-se um software chamado EngineeringEquation Solver (EES). É um programa de fácil manuseio e que facilita os cálculos realizados nas práticas, além de diminuir erros nas contas efetuadas. Para se resolver um cálculo de incerteza é necessário inserir na Janela de Equações (EquationsWindow) as equações utilizadas para que o problema possa ser solucionado.

Com relação ao formato em que as equações devem ser escritas, tem-se que o uso de parênteses é necessário para que o software não interprete erroneamente e assim, para que não se obtenham resultados errados. Caso seja preciso adicionar um comentário ao cálculo, é recomendado o uso de aspas ("), pelo mesmo motivo citado anteriormente.

Ainda, o programa possibilita rearranjar as equações escritas pelo usuário, de modo que fiquem mais organizadas e de fácil entendimento. $\mathrm{O}$ comando a ser utilizado para isso é o "FormattedEquations".

Após esse passo, o próximo é realizar o cálculo da incerteza, de acordo com os valores introduzidos na EquationsWindow. Para isso, é necessário ativar dois comandos no programa EES. O primeiro é o "Check/Format", que faz com que o software cheque as equações e medidas inseridas e verifique se está tudo formatado corretamente, aos padrões do programa.

Depois, utiliza-se o comando "Solve", que irá resolver o problema em questão. Uma nova janela aparecerá com os resultados, sendo que a maioria deles é calculada utilizando o método de arredondamento.

Além do EES, outros programas são utilizados a fim de se calcular a incerteza de uma grandeza ou experimento. Um exemplo é o LCDS, website em que o cálculo do parâmetro trabalho é realizado com facilidade. $\mathrm{O}$ Windows Excel também conta como uma fonte de cálculo, feito por meio de planilhas.

Tendo em vista a necessidade de uma abordagem voltada ao estudo da engenharia, torna-se importante a aplicação de metodologias de projeto para o ensino do cálculo de incertezas, tendo em vista que muitas das metodologias tradicionais demonstram pouca eficácia no que diz respeito ao auxílio que o aluno necessita para desenvolver um pensamento prático eficiente $\mathrm{e}$ autônomo.

Desse modo, a metodologia de projeto é uma abordagem de trabalho que engloba uma relação diferenciada com o conteúdo, mais voltada para a aplicação de conhecimentos em situações reais e culminando em uma formação de competências e aprendizagens práticas.

Baxter (2011) sintetiza os processos de desenvolvimento de uma metodologia em três 
passos básicos: Observação e análise; planejamento e projeção; construção e execução.

No contexto de cálculo das incertezas, os três exemplos seguintes abordam a metodologia de projeto com a finalidade de expor a importância do cálculo de incertezas no campo da engenharia e proporcionar o desenvolvimento do raciocínio matemático.

\subsection{Exemplos práticos como metodologia} didático-pedagógica no ensino de análises de incerteza e sua utilização em estudos de caso em engenharias

Exemplo 1: Dois alunos de engenharia de alimentos, em uma visita a uma cervejaria artesanal, tinham como tarefa realizar parte do controle de qualidade do projeto hidráulicoda indústria e apresentar um memorial descritivo. Para iniciar o projeto os alunos tiveram que determinar o número de Reynolds $(\mathrm{Re})$ da água utilizada na alimentação da unidade de processamento, e a incerteza de associada a essa medição. Como a tubulação já estava instalada, os estudantes utilizaram um barbante para determinar a circunferência da tubulação e, portanto, o diâmetro externo da mesma, quantificando o comprimento da circunferência com uma régua. A determinação da vazão volumétrica $\left(\mathrm{V}_{\mathrm{v}}\right)$ foi realizada através o método gravimétrico utilizando um balde e o cronômetro do celularde um dos estudantes. Sabia-se que a viscosidade dinâmica $(\mu)$ e a densidade $(\rho)$ da água que passava na tubulação eram 0,0089 Pas e $997 \mathrm{Kg} \mathrm{m}^{-3}$, respectivamente. Apresente o memorial de projeto com cálculo do número de Reynolds, a incerteza de medição associada e discussões técnicas.

Exemplo 2: No processo treine de uma empresa foi solicitado aum grupo de engenheiros através de atividade inventiva construíssem um calorímetro alternativo para a empresa. Após construírem o equipamento, o grupo realizouum experimento para determinar a capacidade térmica deste calorímetro para mostrar a empresa à eficácia do equipamento. $\mathrm{O}$ experimento foi realizado com o auxílio de instrumentos como termômetro, balança e termopar. Inicialmente pesou-se certa massa de água à temperatura ambiente $\left(23^{\circ} \mathrm{C}\right)$, que foi em seguida introduzida ao calorímetro. A ação foi realizada novamente, mas dessa vez com água quente $\left(63^{\circ} \mathrm{C}\right)$ e por fim foi medida a temperatura de equilíbrio do sistema $\left(36^{\circ} \mathrm{C}\right)$. Determinar a capacidade térmica do calorímetro junto com a incerteza do equipamento construído.

Exemplo 3: Em um laboratório análise de qualidade de biodiesel, estudantes de engenharia mecânica desejavam quantificar a viscosidade dinâmica $(\mu)$ do óleo de 
cozinharesidual utilizado como matéria prima na transesterificação. O grupo de técnicos adotou o método de Stokes para determinar viscosidade dinâmica do lote de óleo antes da sua utilização no processo. Para a execução do método foi realizado o seguinte experimento: a uma bureta de $50 \mathrm{mLfoi}$ colocada uma régua graduada ao longo do seu comprimento. Posteriormente, a bureta foi enchidacom o óleo de cozinha que se desejava descobrir a viscosidade. Com o auxílio de uma pipeta graduada de $1 \mathrm{~mL}$, foi gotejadoum volume de água destilada sobre o óleo dentro da burete e anotou-se o volume da gota. Considerou-se que a gota de água tinha a geometria de uma esfera, e utilizando a equação do volume da esferafoi determinado o raio da gota. Esperouse a gota se desprender da superfície do óleo (no topo da bureta) e o cronômetro foi acionado, com o objetivo de medir o tempo que a gota de água demorava em percorrer a distância mensurada, calculando, assim, a velocidade. Considerando que a $25^{\circ} \mathrm{C}$ a densidade específica da água $\left(\rho_{1}\right)$ e do óleo usadoeram iguais a $997,2 \mathrm{~kg} \mathrm{~m}^{-3} \mathrm{e} 920,4 \mathrm{~kg} \mathrm{~m}^{-}$ ${ }^{3}$ os estudantes calcularam a viscosidade dinâmica do óleo na temperatura ambiente de $25^{\circ} \mathrm{C}$.

\subsection{Solução do Exemplo 1}

Primeiramente as grandezas que envolviam o experimento devem ser quantificadas. Os valores obtidos para cada grandeza e a incerteza de cada instrumento utilizado na medição estão apresentados na Tabela 1.

Tabela 1- Grandezas do experimento e incertezas dos instrumentos.

\begin{tabular}{cccc}
\hline Grandeza & Unidade & Valor lido & Incertezaassociada a grandeza $\left(\mathrm{w}_{\mathrm{V}} ; \mathrm{w}_{\left.\mathrm{T} ; \mathrm{w}_{\mathrm{D}}\right)}\right.$ \\
\hline Volume $(\mathrm{V})$ & Litro & 25 & 0,0025 \\
\hline Tempo $(\mathrm{T})$ & Segundo & 72,01 & 0,01 \\
\hline Diâmetro (D) & Metro & 0,026 & 0,005
\end{tabular}

$\mathrm{W}_{\mathrm{v}}$ : Incerteza do baldeassociada a medição de volume. $\mathrm{w}_{\mathrm{T}}$ : Incerteza do cronômetro do celularassociada a medição de tempo. $\mathrm{w}_{\mathrm{D}}$ : Incerteza da régua associada a medição de diâmetro.

Passo 1 -Calcular da vazão volumétrica $\left(V_{v}\right)$ através da Equação 4

$$
\mathrm{V}_{\mathrm{V}}=\frac{\mathrm{V}}{\mathrm{T}}(4)
$$

Em que:

$\mathrm{V}_{\mathrm{v}}$ : Vazão volumétrica determinada pelo método gravimétrico $\left(\mathrm{L} \mathrm{s}^{-1}\right)$.

V: Volume de água coletado no balde em um determinado intervalo de tempo (L).

T: Intervalo de tempo utilizado para coletar o volume V (s). 
Os estudantes quantificaram o tempo (T) necessário para coletar no balde, volume (V) de 25 $\mathrm{L}$ de água. Foram realizadas 10 medições obtendo um valor médio para $\mathrm{T}$ de 72,01 s. Portanto aplicando-se a Equação 4 tem-se que a $\mathrm{V}_{\mathrm{V}}$ na tubulação foi de $0,35 \mathrm{~L} \mathrm{~s}^{-1}$.

Passo 2- Aplicar a Equação 3 no cálculo da incerteza propagada associada a medição da vazãovolumétrica $\left(\mathrm{w}_{1}\right)$ gerando a Equação 5.

$\mathrm{w}_{1}=\sqrt{\left(\frac{\partial \mathrm{V}_{\mathrm{v}}}{\partial \mathrm{V}}\right)^{2} *\left(\mathrm{w}_{\mathrm{V}}\right)^{2}+\left(\frac{\partial \mathrm{V}_{\mathrm{v}}}{\partial \mathrm{T}}\right)^{2} *\left(\mathrm{w}_{\mathrm{T}}\right)^{2}}$

Passo 2a: Resolução de $\left(\frac{\partial V_{v}}{\partial V}\right)$

$\left(\frac{\partial \mathrm{V}_{\mathrm{v}}}{\partial \mathrm{V}}\right)=\frac{1}{\mathrm{~T}}=\frac{1}{72,01}$

Passo 2b: Resolução de $\left(\frac{\partial V_{v}}{\partial T}\right)$

$\left(\frac{\partial \mathrm{V}_{\mathrm{v}}}{\partial \mathrm{T}}\right)=\frac{-\mathrm{V}}{\mathrm{T}^{2}}=\frac{-25 * 10^{-3}}{72,01^{2}}$

Passo 2c:Inserindo a resolução de $\left(\frac{\partial V_{v}}{\partial V}\right)$, a resolução de $\left(\frac{\partial V_{v}}{\partial T}\right)$ e os valores de $\mathrm{w}_{\mathrm{V}}$ e $\mathrm{w}_{\mathrm{T}}$ apresentados na Tabela 1 na Equação 5, determina-se o valor de $\mathrm{w}_{1}$ como mostrado abaixo:

$\mathrm{w}_{1}=\sqrt{\left(\frac{1}{72,01}\right)^{2} *(0,0025)^{2}+\left(\frac{-25 * 10^{-3}}{72,01^{2}}\right)^{2} *(0,01)^{2}}=3,5 * 10^{-5} \mathrm{~L} \mathrm{~s}^{-1}$

Portanto a vazão volumétrica da água que escoa na referida tubulação é de $0,35 \pm 3,5 \times 10^{-5} \mathrm{~L} \mathrm{~s}^{-1}$

Passo 3- Calcular a vazão mássica $\mathrm{V}_{\mathrm{m}}$ utilizando a Equação 6

$\mathrm{V}_{\mathrm{m}}=\rho * \mathrm{~V}_{\mathrm{v}}$

$\mathrm{V}_{\mathrm{m}}=997 \frac{\mathrm{Kg}}{\mathrm{m}^{3}} * 0,35 \frac{\mathrm{L}}{\mathrm{s}} * \frac{1 \mathrm{~m}^{3}}{1000 \text { litros }}=0,35 \frac{\mathrm{Kg}}{\mathrm{s}}$

Passo 4- Aplicar a Equação 3 no cálculo da incerteza propagada associada a medição da vazão mássica $\left(\mathrm{w}_{2}\right)$ gerando a Equação 7.

$\mathrm{w}_{2}=\sqrt{\left(\frac{\partial \mathrm{V}_{\mathrm{m}}}{\partial \mathrm{V}_{\mathrm{v}}}\right)^{2} *\left(\mathrm{w}_{1}\right)^{2}}$ 
Passo 4a: Resolução de $\left(\frac{\partial \mathrm{V}_{\mathrm{m}}}{\partial \mathrm{V}_{\mathrm{v}}}\right)$

$\left(\frac{\partial \mathrm{V}_{\mathrm{m}}}{\partial \mathrm{V}_{\mathrm{v}}}\right)=\rho=997$

Passo 4b:Inserindona Equação 7a resolução de $\left(\frac{\partial \mathrm{V}_{\mathrm{m}}}{\partial \mathrm{V}_{\mathrm{v}}}\right)$ e o valor de $\mathrm{w}_{1}$ determinado no Passo 2c, calcula-se o valor de $\mathrm{w}_{2}$ apresentado abaixo:

$\mathrm{w}_{2}=\sqrt{(\rho)^{2} *\left(3,47 * 10^{-5}\right)^{2}}=3,5 * 10^{-2}$

Portanto, a vazão mássica da água que escoa na referida tubulação é de $0,35 \pm 3,5 \times 10^{-2} \mathrm{Kg} \mathrm{s}^{-1}$

Passo 5 - Cálculo do número de Reynolds utilizando a Equação 8.

$\mathrm{Re}=\frac{\mathrm{V}_{\mathrm{m}} * \mathrm{D}}{\mu * \mathrm{~A}}=\frac{4 * \mathrm{~V}_{\mathrm{m}}}{\pi * \mu * \mathrm{D}}$

$\operatorname{Re}=\frac{4 * 0,346}{\pi * 0,0089 * 0,026}=1,9 * 10^{4}$

Passo 6- Aplicar a Equação 3 no cálculo da incerteza propagada associada a determinação do número de Reynolds (w3) gerando a Equação 9.

$w=\sqrt{\left(\frac{\partial R e}{\partial V_{m}}\right)^{2} *\left(w_{2}\right)^{2}+\left(\frac{\partial R e}{\partial D}\right)^{2} *\left(w_{D}\right)^{2}}$

Passo 6a: Resolução de $\left(\frac{\partial R e}{\partial V_{m}}\right)$

$\left(\frac{\partial R e}{\partial V_{m}}\right)=\frac{4}{\pi * \mu * D}=5502,33$

Passo 6b: Resolução de $\left(\frac{\partial R e}{\partial D}\right)$

$\left(\frac{\partial R e}{\partial D}\right)=\frac{-4 * V_{m}}{\pi * \mu * D^{2}}=73223,34$

Passo 6c:Inserindona Equação 9as resoluções de $\left(\frac{\partial R e}{\partial V_{m}}\right)$ e $\left(\frac{\partial R e}{\partial D}\right)$, o valor de $\mathrm{w}_{2}$ determinado no Passo $4 \mathrm{~b}$ e valor de $\mathrm{w}_{\mathrm{D}}$ apresentado na Tabela 1,calcula-se o valor de $\mathrm{w}_{3}$ como mostrado abaixo: $w_{3}=\sqrt{(5502,33)^{2} *\left(3,46 * 10^{-2}\right)^{2}+(73223,34)^{2} *(0,0005)^{2}}=193,9$

Portanto, o número de Reynolds da água que escoa na referida tubulação é de $19000 \pm$ 194. Assim é possível concluir a metodologia utilizada pelos estudantes proporcionou o cálculo do valor de Re com o erro de $1 \%$. 
Analisando a marcha de cálculo utilizada para determinar o valor da incerteza associada ao cálculo do número de Reynolds $\left(w_{3}\right)$ é possível afirmar que a propagação de incertezas na determinação do Re, é provocada principalmentepor erros ocorridos na medição do diâmetro da tubulação, isso porque esse é o fator com o maior valor de derivada dentre os demais utilizados na determinação de $w_{3}$. Como a circunferência da tubulação foi determinada com um barbante e o diâmetro externo calculado através desta, é possível afirmar que não se deve utilizar o diâmetro externo de uma tubulação para cálculo do número de $\mathrm{Re}$, pois isso contribuiu para uma grande imprecisão na medida.

Ao utilizar o barbante para quantificação da circunferência da tubulação foram constatados erros instrumentais e observacionais, respectivamente. Para diminuir tais erros é necessárioa utilização de instrumentos calibrados e apropriados para realizar as medidas necessárias como paquímetros, além de atenção no manuseio e leitura dos instrumentos.

\subsection{Solução do Exemplo 2}

Primeiramente devem ser determinadas as grandezas que envolvem o experimento, os valores obtidos para cada grandeza e a incerteza de cada instrumento utilizado na determinação de cada grandeza como mostrado na Tabela 2.

Tabela 2-Grandezas registradas no experimento e incerteza de cada instrumento utilizado na determinação da grandeza

\begin{tabular}{cccc}
\hline Grandeza & Unidade & Valor lido & Incerteza $\left(\mathrm{w}_{4} ; \mathrm{w}_{5} ; \mathrm{w}_{6} ; \mathrm{w}_{7}\right)$ \\
\hline Temperatura da água em condições ambientais & ${ }^{\circ} \mathrm{C}$ & 23 & $1{ }^{\circ} \mathrm{C}$ \\
Temperatura da água aquecida & ${ }^{\circ} \mathrm{C}$ & 63 & $1{ }^{\circ} \mathrm{C}$ \\
Temperatura do equilíbrio & ${ }^{\circ} \mathrm{C}$ & 36 & $1{ }^{\circ} \mathrm{C}$ \\
Massa de água aquecida & $\mathrm{G}$ & 11,20 & $0,001 \mathrm{~g}$ \\
Massa de água fria & $\mathrm{G}$ & 11,65 & $0,001 \mathrm{~g}$ \\
\hline
\end{tabular}

$\mathrm{w}_{4}$ : incerteza do termômetro relacionada à massa da água em temperatura ambiente. $\mathrm{w}_{5}$ : incerteza do termômetro relacionada a temperatura da água em condição ambiente $\left(23^{\circ} \mathrm{C}\right) \cdot \mathrm{w}_{6}$ : incerteza do termômetro relacionada a temperatura da água aquecida $\left(63^{\circ} \mathrm{C}\right)$. $\mathrm{w}_{7}$ :incerteza da balança relacionada à massa da água.

Passo 1 - Calcular a capacidade térmica de um calorímetro construído (C) através da Equação 12 obtida a partir da operação matemática das equações 10 e 11 .

$$
\begin{gathered}
\mathrm{Q}_{\text {calorímetro }}+\mathrm{Q}_{\text {águanatural }}+\mathrm{Q}_{\text {águaquente }}=0 \\
\text { Revista da Universidade Vale do Rio Verde, Três Corações, v. 14, n. 2, p. 1136-1151, ago./dez. } 2016
\end{gathered}
$$


$\mathrm{Cx}\left(\mathrm{T}_{\text {eq }}-\mathrm{T}_{0}\right)+\mathrm{m}_{\text {águ a natural }} \mathrm{x}_{\mathrm{p}} \mathrm{x}\left(\mathrm{T}_{\text {eq }}-\mathrm{T}_{0}\right)+\mathrm{m}_{\text {água quente }} \mathrm{x}_{\mathrm{P}} \mathrm{x}\left(\mathrm{T}_{\text {eq }}-\mathrm{T}_{0^{\prime}}\right)=0$

$\mathrm{C}=\frac{-\mathrm{m}_{1} \times \mathrm{c}_{\mathrm{p}} \times\left(\mathrm{T}_{\mathrm{eq}}-\mathrm{T}_{0}\right)+\mathrm{m}_{2} \times \mathrm{c}_{\mathrm{P}} \times\left(\mathrm{T}_{\mathrm{eq}}-\mathrm{T}_{0^{\prime}}\right)}{\left(\mathrm{T}_{\mathrm{eq}}-\mathrm{T}_{0}\right)}$

Em que:

C: Capacidade térmica do calorímetro construído $\left(\right.$ cal $\left.^{\circ} \mathrm{C}^{-1}\right)$.

$\mathrm{m}_{1}$ : massa da água a temperatura de $23^{\circ} \mathrm{C}(\mathrm{g})$.

$\mathrm{m}_{2}$ : massa da água a temperatura de $63^{\circ} \mathrm{C}(\mathrm{g})$.

$T_{0^{\prime}}$ : Temperatura da água quente $\left({ }^{\circ} \mathrm{C}\right)$

$T_{0}:$ Temperatura da água natural $\left({ }^{\circ} \mathrm{C}\right)$

$\mathrm{c}_{\mathrm{p}}$ : calor específico da água $\left(1 \mathrm{cal} \mathrm{g}^{-1} \mathrm{C}^{-1}\right)$

$C=\frac{-11,65 \times 1 \times(36-23)-11,20 \times 1 \times(36-63)}{36-23}$

$C=11,61 \frac{\mathrm{cal}}{{ }^{\circ} \mathrm{C}}$

Passo 2-Aplicar a Equação 3 no cálculo da incerteza associadaa capacidade térmica do calorímetro construído $\left(\mathrm{w}_{\mathrm{C}}\right)$ gerando a Equação 13.

$\mathrm{w}_{\mathrm{C}}=\sqrt{\left(\frac{\partial \mathrm{C}}{\partial \mathrm{m}_{1}}\right)^{2}\left(\mathrm{w}_{7}\right)^{2}+\left(\frac{\partial \mathrm{C}}{\partial\left(\mathrm{T}_{\mathrm{eq}}-\mathrm{T}_{0}\right)}\right)^{2} *\left(\mathrm{w}_{5}\right)^{2}+\left(\frac{\partial \mathrm{C}}{\partial\left(\mathrm{T}_{\mathrm{eq}}-\mathrm{T}_{0}^{\prime}\right)}\right)^{2} *\left(\mathrm{w}_{6}\right)^{2}}$

Passo 2a: Resolução da sentença $\left(\frac{\partial C}{\partial m_{1}}\right)$

$\frac{\partial \mathrm{C}}{\partial \mathrm{m}_{1}}=-\mathrm{c}_{\mathrm{p}}=-4,2$

Passo 2b: Resolução da sentença $\left(\frac{\partial \mathrm{C}}{\partial\left(\mathrm{T}_{\mathrm{eq}}-\mathrm{T}_{0}\right)}\right)$

$\frac{\partial \mathrm{C}}{\partial\left(\mathrm{T}_{\mathrm{eq}}-\mathrm{T}_{0}\right)}=\frac{\mathrm{m}_{2} \times \mathrm{c}_{\mathrm{P}}}{\left(\mathrm{T}_{\mathrm{eq}}-\mathrm{T}_{0}\right)}=3,62 \times 10^{-3}$

Passo 2c: Resolução da sentença $\left(\frac{\partial \mathrm{C}}{\partial\left(\mathrm{T}_{\mathrm{eq}}-\mathrm{T}_{0}^{\prime}\right)}\right)$

$\frac{\partial \mathrm{C}}{\partial\left(\mathrm{T}_{\mathrm{eq}}-\mathrm{T}_{0}{ }^{\prime}\right)}=\frac{-\mathrm{m}_{2} \times \mathrm{c}_{\mathrm{P}} \times\left(\mathrm{T}_{\mathrm{eq}}-\mathrm{T}_{0}{ }^{\prime}\right)}{\left(\mathrm{T}_{\mathrm{eq}}-\mathrm{T}_{0}\right)^{2}}=7,51 \times 10^{-3}$

$\mathrm{w}_{\mathrm{C}}=\sqrt{(-4,2)^{2} \times\left(10^{-3}\right)^{2}+\left(3,62 \times 10^{-3}\right)^{2} \times(1)^{2}+\left(7,51 \times 10^{-3}\right)^{2} \times(1)^{2}}=1,115 \times 10^{-5}$ 
Portanto, o calorímetro construído

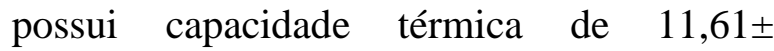
$0,000011 \mathrm{cal}^{\circ} \mathrm{C}^{-1}$. Assim é possível concluir que o calorímetro construído não é adiabático "perfeito", permitindo um erro de $10^{-5} \%$ no processo.

Analisando a marcha de cálculo utilizada para determinar o valor da incerteza propagada associada ao cálculo da capacidade térmica do calorímetro construído $\left(\mathrm{w}_{\mathrm{C}}\right)$ é possível afirmar que a propagação de incertezas na determinação do C, é provocada principalmentepela relação entre a capacidade calorifica e massa de água (Passo $2 \mathrm{~b}$ do item 2.6) sendo que o item agravante foi a incerteza vinculada a massa de água. Assim, diminuir os erros obtidos na balança seria uma alternativa para diminuir a incerteza associada ao procedimento.

Todavia, é de suma importância ressaltar que as atividades realizadas acarretaram em erros de certos tipos, visto que a cada etapa do procedimento ocorreu autilização de um instrumento ou foi executada uma ação que agrega erros. Primeiramente, com a balança, teve-se os erros instrumentais, ocasionados por uma possibilidade de calibração incorreta da mesma. Acrescidos a estes estão os erros ambientais, que no procedimento em questão foram devidos à ausência de condições controladas.

Os erros observacionais, atribuídos à uma visualização inapropriada das temperaturas no termômetrotambém estãopresentes. Finalmente, ocorreram os erros teóricos, de ocorrência frequente quando são utilizados fórmulas e procedimentos matemáticos.As soluções para minimizar os erros anteriormente descritos estão muitas vezes implícitas na própria fonte de erro, como evitar realizar a ação que conduz ao mesmo e agregar ao experimento condições controladas que visem a uma redução da influência do ambiente externo nos procedimentos.

\subsection{Solução do Exemplo 3}

Primeiramente devem ser determinadas as grandezas que envolvem o experimento, os valores obtidos para cada grandeza e a incerteza de cada instrumento utilizado na determinação de cada grandeza como mostrado na Tabela 3.

Tabela 3- Grandezas registradas no experimento e incerteza de cada instrumento utilizado na determinação da grandeza

\begin{tabular}{ccc}
\hline Grandeza & Unidade & Valor lido \\
\hline Revista da Universidade Vale do Rio Verde, Três Corações, v. 14, n. 2, p. 1136-1151, ago./dez. 2016
\end{tabular}




\begin{tabular}{cccc}
\hline Raio & $\mathrm{m}$ & 0,003296 & 0,0007325 \\
Velocidade & $\mathrm{m} \mathrm{s}^{-1}$ & 0,012402 & 0,001752 \\
$\rho$ & $\mathrm{Kg} \mathrm{m}^{-3}$ & 920,4 & 9,213 \\
\hline
\end{tabular}

W8: incerteza relacionada ao cálculo do raio da esfera. W9: $_{9}$ incerteza ao cálculo da velocidade de percurso da esfera. $\mathrm{w}_{10}$ : incerteza relacionada ao cálculo densidade da água ou do óleo (massa/volume).

Passo 1 -Determinar o raio da gota de água utilizando a Equação 14.

$V_{g}=\frac{4 \pi R^{3}}{3}$

Em que:

Vg: Volume da gota de água quantificado pelo volume retirado na pipeta graduada a cada gota $(\mathrm{mL})$.

R: Raio da esfera de água gotejada dentro da bureta com óleo $(\mathrm{cm})$.

Como cada gota de água tinha o volume de aproximadamente $0,15 \mathrm{~cm}^{3}$, após usar a Equação 14 foi determinado que o raio médio da esfera era de $0,33 \mathrm{~cm}$.

Passo 2 - Determinar a velocidade com que a gota percorre a bureta (V).

A gota gastava aproximadamente 24,19 segundos para percorrer $30 \mathrm{~cm}$, portanto a velocidade de percurso da gota era de $1,2402 \mathrm{~cm} \mathrm{~s}^{-1}$.

Passo 3-Calcular a viscosidade dinâmica do óleo de cozinha usado $(\mu)$ através da Equação 15.

$$
\mu=\frac{2 *\left(\rho_{1}-\rho_{2}\right) * g * \mathrm{R}^{2}}{9 * \mathrm{~V}}
$$

Em que :

$\mu$ : Viscosidade dinâmica do óleo de cozinha usado (Pa s).

$\rho_{1}$ : Densidade específica da água utilizada para gerar a gota $\left(\mathrm{Kg} \mathrm{m}^{-3}\right)$.

$\rho_{2}$ : Densidade específica do óleo usado $\left(\mathrm{Kg} \mathrm{m}^{-3}\right)$.

$\mathrm{g}$ : Aceleração da gravidade $\left(\mathrm{m} \mathrm{s}^{-2}\right)$.

R: Raio da esfera de água (m).

v: velocidade de percurso da gota de água $\left(\mathrm{m} \mathrm{s}^{-1}\right)$.

$\mu=\frac{2 *(997,2-920,4) * 10 * 0,003296^{2}}{9 * 0,012402}=0,1495$ Pa s 
Passo 4-Aplicação da Equação 3 para o cálculo da incerteza associada ao cálculo daviscosidade dinâmica do óleo de cozinha $\left(\mathrm{w}_{\mu}\right)$ gerando a Equação 16.

$\mathrm{w}_{\text {回 }}=\sqrt{\left(\frac{\partial \mu}{\partial \mathrm{R}}\right)^{2} *\left(\mathrm{w}_{\mathrm{R}}\right)^{2}+\left(\frac{\partial \mu}{\partial \rho_{2}}\right)^{2} *\left(\mathrm{w}_{\rho_{2}}\right)^{2}\left(\frac{\partial \mu}{\partial \mathrm{v}}\right)^{2} *\left(\mathrm{w}_{\mathrm{v}}\right)^{2}}$

Em que:

Passo 2a: Resolução da sentença $\left(\frac{\partial \mu}{\partial R}\right)$

$\left(\frac{\partial \mu}{\partial \mathrm{R}}\right)=\frac{4 *\left(\rho_{1}-\rho_{2}\right) * \mathrm{~g} * \mathrm{R}}{9 * \mathrm{v}}=90,71$

Passo 2b: Resolução da sentença $\left(\frac{\partial \mu}{\partial \rho_{2}}\right)$

$\left(\frac{\partial \mu}{\partial \rho_{2}}\right)=\frac{-2 * g * \mathrm{R}^{2}}{9 * \mathrm{~V}}=-0,001947$

Passo 2c: Resolução da sentença $\left(\frac{\partial \mu}{\partial v}\right)$

$\left(\frac{\partial \mu}{\partial v}\right)=\frac{-2 *\left(\rho_{1}-\rho_{2}\right) * g * \mathrm{R}^{2}}{9 * \mathrm{v}^{2}}=-12,05$

$w_{\text {曰 }}=\sqrt{(90,71)^{2} *(0,0007325)^{2}+(-0,001947)^{2} *(9,213)^{2}(-12,05)^{2} *(0,001752)^{2}}$

$w_{\text {曰] }}=0,07199$

Após o experimento é possível concluir que a viscosidade dinâmica média do lote de óleo de cozinha usado que foi analisado é de $0,15 \pm 0,072 \mathrm{~Pa}$ s.

Uma vez que a derivada da viscosidade em relação ao raio possui o maior valor em relação entre as parcelas utilizadas no cálculo de $w_{\mu}$, conclui-se que erros na determinação do raio representam o fator que mais influencia na incerteza do experimento. Esses erros sistemáticos do tipo instrumentais e observacionais podem ter sido causados pela má calibração ou desgaste da marcação na pipeta e/ou de falhas de procedimento do observador devido ao efeito de paralaxe. Tais erros podem ser minimizadosutilizando uma pipeta nova e seguindo cuidadosamente os procedimentos corretos de alinhamento entre o olho do observador (nivelamento do menisco), o indicador da leitura e a escala do instrumento.

Além destes erros, pode ter ocorrido ainda o erro do tipo teórico, uma vez que, após o cálculo do raio, possivelmente foi feito um arredondamento. Este tipo de erro pode ser reduzido utilizando-se modelos físicos e valores suficientemente exatos para o experimento em questão. 


\section{CONSIDERAÇÕES FINAIS}

O conceito de incerteza como um atributo quantificável é relativamente novo na história da medição, embora o conceito de erro e análise de erro tenham sido, há muito, uma parte da prática da ciência da medição ou metrologia.

Mesmo quando todos os componentes de erro conhecidos ou presumidos tenham sido avaliados e as correções adequadas tenham sido aplicadas, ainda permanece uma incerteza sobre quão correto é o resultado declarado, isto é, uma dúvida acerca de quão corretamente o resultado da medição representa o valor da grandeza que está sendo medida.

Da mesma forma como o uso quase universal do Sistema Internacional de Unidades (SI) trouxe coerência a todas as medições científicas e tecnológicas, um consenso mundial sobre a avaliação e expressão da incerteza de medição permitiria que o significado de um vasto espectro de resultados de medições na ciência, engenharia, comércio, indústria e regulamentação, fosse prontamente compreendido e apropriadamente interpretado. Nesta era de mercado global, é imperativo que o método para avaliar e expressar incerteza seja uniforme em todo o mundo, de forma tal que as medições realizadas em diferentes países possam ser facilmente comparada.

\section{REFERÊNCIAS}

BARATTO, A. C.; DAMASCENO, J. C.; ALVES, J. A. P.; TROTA FILHO, J.; COUTO, P. R. G.; OLIVEIRA, S. P.Avaliação de dados de medição — Guia para a expressão de incerteza de medição.Editora do Grupo de Trabalho do Comitê Conjunto para Guias em Metrologia (JCGM/WG), 2008. 138p.

BAXTER, M. Projeto de Produto. São Paulo: Edgard Blücher, 2011.

CAMPOS, A. A. G.; ALVES, E. S.; SPEZIALI, N. L.. Física experimental básica na universidade. $2^{\mathrm{a}}$ edição revista Belo Horizonte : Editora UFMG, 2008. 213p.

MILLS, A. F. Error analysis of experiments.University of California - Los Angeles, California, 2004.40p.

SANTOS JÚNIOR, M. J.;IRIGOYEN, E. R. C. Metrologia dimensional: teoria e prática. $1^{\text {a }}$ edição - Porto Alegre : Editora da Universidade/UFRGS, 1985. 190p.

SILVA NETO, J.C. Metrologia e controle dimensional. - Rio de Janeiro :Elsevier, 2012.

VUOLO, A. F.. Fundamentos da teoria de erros.São Paulo. EdgardBlücher, 2011. 